\title{
ORIGINAL
}

\section{Análisis de 208 fibrobroncoscopias realizadas en una unidad de cuidados intensivos}

\section{Á. Estella}

Servicio de Medicina Intensiva, Hospital SAS de Jerez, Jerez de la Frontera, Cádiz, España

Recibido el 2 de agosto de 2011; aceptado el 4 de noviembre de 2011

Disponible en Internet el 20 de diciembre de 2011

\author{
PALABRAS CLAVE \\ Fibrobroncoscopia; \\ Unidad de Cuidados \\ Intensivos; \\ Neumonía; \\ Atelectasias; \\ Lavado \\ broncoalveolar
}

\begin{abstract}
Resumen
Objetivo: Describir las principales indicaciones, resultados clínicos y complicaciones de la fibrobroncoscopia en enfermos críticos.

Diseño: Estudio retrospectivo, observacional, de un solo centro.

Ámbito: Unidad de Cuidados Intensivos (UCI) médico-quirúrgica de 17 camas.

Pacientes: Pacientes consecutivos a los que se les realizó una fibrobroncoscopia durante un periodo de cinco años.

Intervenciones: Fibrobroncoscopia realizada por médicos especialistas en Medicina Intensiva con fines diagnósticos y/o terapéuticos.

Principales variables de interés: Indicaciones y complicaciones derivadas de la fibrobroncoscopia.

Resultados: Se han realizado 208 fibrobroncoscopias en 192 pacientes; en el momento del procedimiento 193 (92,8\%) recibían ventilación mecánica invasiva. La edad media de los pacientes incluidos fue de $58 \pm 16$ años y el APACHE II al ingreso en UCI de $19 \pm 7$. La mortalidad global fue del $31,3 \%$. Las indicaciones más frecuente fueron en $148(71,2 \%)$ casos por sospecha clínica de neumonía y en $28(13,5 \%)$ para resolución de atelectasias. La fibrobroncospia fue eficaz en $120(57,7 \%)$ casos, con resolución de la atelectasia en 20 casos, 71,4\% y obteniendo resultados positivos del LBA en 68 (46\%) de los casos con sospecha de neumonía. Se han detectado 27 complicaciones menores en 208 (13\%) pacientes. Las complicaciones más frecuentes han sido: taquicardia supraventricular $(3,8 \%)$, hipoxemia transitoria $(6,7 \%)$ y hemorragia leve de la mucosa bronquial $(2,4 \%)$.

Conclusiones: El diagnóstico microbiológico de neumonías y la resolución de atelectasias fueron las indicaciones más frecuentes. La fibrobroncoscopia realizada por especialistas de Medicina Intensiva es un procedimiento eficaz y seguro.

(c) 2011 Elsevier España, S.L. y SEMICYUC. Todos los derechos reservados.
\end{abstract}

Correo electrónico: litoestella@hotmail.com 


\section{KEYWORDS \\ Flexible \\ bronchoscopy; \\ Intensive Care Unit; \\ Pneumonia; \\ Atelectasis; \\ Bronchoalveolar \\ lavage}

\section{Analysis of 208 flexible bronchoscopies performed in an intensive care unit}

\begin{abstract}
Objective: To describe the main indications, clinical results and complications associated with fibrobronchoscopy in the Intensive Care Unit (ICU).

Design: A retrospective, single-center observational study was carried out.

Setting. Seventeen beds in a medical/surgical ICU.

Patients. Consecutive patients undergoing fibrobronchoscopy during their stay in the ICU over a period of 5 years.

Interventions: Flexible bronchoscopy performed by an intensivist.

Main variables of interest. Flexible bronchoscopy indications and complications derived from the procedure.

Results: A total of 208 flexible bronchoscopies were carried out in 192 patients admitted to the ICU. Most of the procedures (193 [92.8\%]) were performed in mechanically ventilated patients. The average patient age was $58 \pm 16$ years, with an APACHE II score at admission of $19 \pm 7$. The most frequent indication for flexible bronchoscopy was diagnostic confirmation of initially suspected pneumonia (148 procedures), with positive bronchoalveolar lavage findings in $46 \%$. The most frequent therapeutic indication was the resolution of atelectasis (28 procedures). Other indications were the diagnosis and treatment of pulmonary hemorrhage, the aspiration of secretions, control of percutaneous tracheotomy, and difficult airway management. The complications described during the procedures were supraventricular tachycardia (3.8\%), transient hypoxemia (6.7\%), and slight bleeding of the bronchial mucosal membrane $(2.4 \%)$. Conclusions: A microbiological diagnosis of pneumonia and the resolution of atelectasis are the most frequent indications for flexible bronchoscopy in critically ill patients.

Flexible bronchoscopy performed by an intensivist in ICU is a safe procedure. (c) 2011 Elsevier España, S.L. and SEMICYUC. All rights reserved.
\end{abstract}

\section{Introducción}

La fibrobroncoscopia se ha convertido en el procedimiento de elección en la mayoría de las exploraciones de la vía aérea y es un medio de ayuda importante en el diagnóstico y tratamiento de varias enfermedades pulmonares en pacientes $\operatorname{críticos}^{1-4}$. La expansión de su uso en las unidades de cuidados intensivos ( $\mathrm{UCl}$ ) se debe a que es una técnica relativamente fácil de realizar a pie de cama ${ }^{2}$, que evita desplazamientos potencialmente peligrosos fuera de la $\mathrm{UCI}$ y con la que se han descrito escasas complicaciones ${ }^{5}$, de tal forma, que actualmente se considera un instrumento esencial dentro del equipamiento de una $\mathrm{UCI}^{5,6}$.

Aunque en principio los pacientes críticos están más predispuestos a desarrollar complicaciones durante este procedimiento, los enfermos en ventilación mecánica, por el hecho de tener asegurada la vía aérea, tienen paradójicamente menor riesgo que cuando esta se realiza en enfermos en ventilación espontánea. El objetivo del presente estudio es describir las principales indicaciones, resultados clínicos, efectividad del lavado broncoalveolar y complicaciones de la fibrobroncoscopia en enfermos críticos.

\section{Pacientes y métodos}

Estudio retrospectivo, observacional de un solo centro, realizado en una UCl médico-quirúrgica dotada de una unidad polivalente de 10 camas y una unidad coronaria de 7 camas. Se incluyeron pacientes consecutivos a los que se les realizó una fibrobroncoscopia estando ingresados en UCl durante un periodo de 5 años. Se analizaron las siguientes características clínicas: edad, APACHE II al ingreso, mortalidad e indicaciones de fibrobroncoscopia así como las complicaciones derivadas de la técnica. No se les realizó la exploración a aquellos enfermos cuya situación clínica y/o hemodinámica no garantizaba la realización de la fibrobroncoscopia con seguridad. Los criterios de exclusión fueron los siguientes: tubo endotraqueal menor de $8 \mathrm{~mm}$ de diámetro, existencia de neumotórax en la radiografía de tórax previa a la realización de la fibrobroncoscopia, saturación de oxígeno, determinada mediante pulsioximetría, menor de $90 \%$ con $\mathrm{FIO}_{2}$ de 1 , acidosis severa $(\mathrm{pH}<7,20)$, inestabilidad hemodinámica definida por tensión arterial sistólica $<90 \mathrm{mmHg}$ a pesar de fármacos vasoactivos.

Durante el procedimiento se realizó monitorización electrocardiográfica continua mediante monitores de cabecera Datex-Ohmeda S/5. La tensión arterial se midió de forma no invasiva mediante el módulo M-NE12STPR y en los casos en que esta era menor de $90 \mathrm{mmHg}$ se procedió a comprobar mediante manguito manual y esfingomanómetro. En función del motivo de ingreso en UCl los pacientes se clasificaron en quirúrgicos (pacientes ingresados en $\mathrm{UCl}$ procedentes de quirófano o áreas quirúrgicas tras una intervención reciente), neurológicos (enfermos ingresados en $\mathrm{UCl}$ por enfermedad del sistema nervioso central), traumatológicos (ingresados en $\mathrm{UCl}$ tras presentar traumatismo grave), inmunodeprimidos (pacientes con alteraciones del sistema inmunitario) y médicos (aquellos procedentes del área de Urgencias o planta de hospitalización de especialidades médicas). 
Previo consentimiento informado verbal y obtenido por escrito se realizó el procedimiento con un broncoscopio modelo Olympus type $40 / 240$ conectado a una fuente de luz halógena Olympus CLK-4. En los enfermos con ventilación mecánica se realizó a través del tubo orotraqueal mediante una válvula adaptadora especial, modelo Mallinckrodt 331/5661. El modo de ventilación durante el procedimiento fue: presión control con $\mathrm{FIO}_{2}$ de 1 y sin efectuar cambios en la presión tele-espiratoria (PEEP) previa. Tras el procedimiento la $\mathrm{FIO}_{2}$ fue ajustada a la situación clínica del enfermo. En cuanto a la sedación utilizada, en los enfermos en ventilación mecánica no se modificó la infusión continua que ya recibían, utilizándose dosis adicionales de midazolam (bolos intravenosos de $1-3 \mathrm{mg}$ ) si las condiciones del paciente lo requerían; en los pacientes en ventilación espontánea se utilizó lidocaína administrada localmente en nariz y orofaringe así como intratraqueal con inyección directa mediante punción intercricotiroidea. En el grupo de enfermos con sospecha clínica de neumonía se les realizó lavado broncoalveolar (BAL) con $150 \mathrm{ml}$ de suero salino fisiológico, repartidos en tres alícuotas de $50 \mathrm{ml}$ e instilados en la localización sugerida por la radiografía de tórax o a nivel de lóbulo medio en el caso de infiltrado bilateral. No se administraron anestésicos locales a través del canal del broncoscopio y se evitó la aspiración hasta enclavar el mismo en la localización a estudiar. Al realizar el BAL, los primeros $20 \mathrm{ml}$ recuperados fueron desechados y una muestra del líquido restante fue procesada para estudio microbiológico consistente en tinción directa de la muestra (tinción de Gram), tinción para micobacterias y hongos, cultivo bacteriano en los medios habituales (agar sangre, agar chocolate, MacConkey, agar Brucella y $\mathrm{BHI}$ ) y en medios específicos para Mycobacteria (medio líquido: Bactec MGIT960-BD), hongos (agar Sabouraud) y Legionella (BCYE). En enfermos inmunodeficientes se realizó tinción específica para Pneumocystis jirovecii. También se aplicaron técnicas de inmunofluorescencia para Legionella. No se realizó cuantificación de células epiteliales y neutrófilos como control de calidad de la muestra. El BAL se consideró positivo ${ }^{7}$ con crecimiento de más de $10^{4}$ unidades formadoras de colonia $/ \mathrm{ml}$ ( ufc/ml). Se realizó radiografía de tórax tras el procedimiento para evaluar la presencia de neumotórax. Las complicaciones se clasificaron en mayores o menores en función de la necesidad de aplicar algún tratamiento y según la guía de la Sociedad Torácica Británica sobre broncoscopia diagnóstica ${ }^{6}$, se definió taquicardia como ritmo cardíaco a una frecuencia superior a 100 latidos por minuto, hipoxemia como saturación de oxígeno menor de $90 \%$ con $\mathrm{FIO}_{2}$ de 1 , y el sangrado por visualización directa a través del broncoscopio durante el procedimiento. Todos los procedimientos fueron realizados por intensivistas, tras haberse formado previamente en la realización de la técnica bajo supervisión en el Servicio de Neumología.

Los datos recogidos fueron analizados mediante el programa estadístico SPSS versión 18 para Windows. Las variables cualitativas se describen con el porcentaje de distribución de cada una de las categorías. Las variables cuantitativas se describen con la media y la desviación estándar cuando siguen una distribución normal, con la mediana en caso contrario.
Tabla 1 Características de los enfermos analizados en el estudio

\begin{tabular}{lll}
\hline $\begin{array}{l}\text { Total } \\
\mathrm{n}=208\end{array}$ & $\begin{array}{l}\mathrm{VMI} \\
\mathrm{n}=193\end{array}$ & $\begin{array}{l}\text { No VMI } \\
\mathrm{n}=15\end{array}$ \\
\hline Edad, años & $58,2 \pm 16,5$ & $53,6 \pm 17,6$ \\
APACHE II al ingreso en UCI & $19,6 \pm 6,8$ & $11,6 \pm 5$ \\
Diagnóstico al ingreso (\%) & & \\
$\quad$ Médico & 57,5 & 66,7 \\
Quirúrgico & 10,9 & 0 \\
$\quad$ Trauma & 8,8 & 6,7 \\
$\quad$ Neurológico & 8,8 & 0 \\
Inmunodeprimidos & 14 & 26,7 \\
Mortalidad & 32,1 & 20 \\
\hline
\end{tabular}

Datos presentados con media \pm desviación estándar. No VMI: sin ventilación mecánica invasiva; VMI: ventilación mecánica invasiva.

\section{Resultados}

Durante el tiempo de estudio el número de ingresos medios anuales fue de 1.182 enfermos, con una media de 357 $(30,2 \%)$ pacientes en ventilación mecánica al año. Un total de 208 fibrobroncoscopias realizadas a 192 pacientes fueron analizadas, lo que corresponde a un 3,51\% del total de los enfermos y un $11,65 \%$ de los enfermos que precisaron conexión a ventilación mecánica.

En $15(7,2 \%)$ casos las broncoscopias se realizaron en pacientes en ventilación espontánea o con ventilación mecánica no invasiva modo BIPAP y en 193 (92,8\%), se hicieron en enfermos con ventilación mecánica. Las características clínicas de los pacientes incluidos, dependiendo de si la exploración se realizó en ventilación mecánica o no, se exponen en la tabla 1. Las principales indicaciones de la fibrobroncoscopia están expuestas en la tabla 2, destacando las indicaciones con finalidad diagnóstica, en especial la sospecha de neumonía, en la que se obtuvo un resultado positivo de los cultivos microbiológicos en $70(46,4 \%)$ casos,

Tabla 2 Indicaciones de fibrobroncoscopia en los enfermos incluidos en el estudio

\begin{tabular}{llr}
\hline $\begin{array}{l}\text { Total } \\
\mathrm{n}=208\end{array}$ & $\begin{array}{l}\text { VMI } \\
\mathrm{n}=193\end{array}$ & $\begin{array}{l}\text { No VMI } \\
\mathrm{n}=15\end{array}$ \\
\hline $\begin{array}{l}\text { Sospecha clínica de } \\
\text { neumonía }\end{array}$ & $140(72,5 \%)$ & $8(53,3 \%)$ \\
$\begin{array}{l}\text { Atelectasias } \\
\text { Hemorragia pulmonar }\end{array}$ & $28(14,5 \%)$ & 0 \\
$\begin{array}{l}\text { Aspiración secreciones } \\
\quad \text { broncorrea) }\end{array}$ & $6(4,1 \%)$ & 0 \\
$\begin{array}{l}\text { Control traqueostomía } \\
\text { Estudio }\end{array}$ & $5(2,6 \%)$ & 0 \\
$\quad$ anatomopatológico & $3(1,6 \%)$ & $2(13,3 \%)$ \\
Vía aérea difícil & $0(0 \%)$ & $2(13,3)$ \\
Otros & $3(1,6 \%)$ & $3(20,1 \%)$ \\
\hline
\end{tabular}

Datos presentados con frecuencias absolutas y relativas (porcentajes).

No VMI: sin ventilación mecánica invasiva; VMI: ventilación mecánica invasiva. 


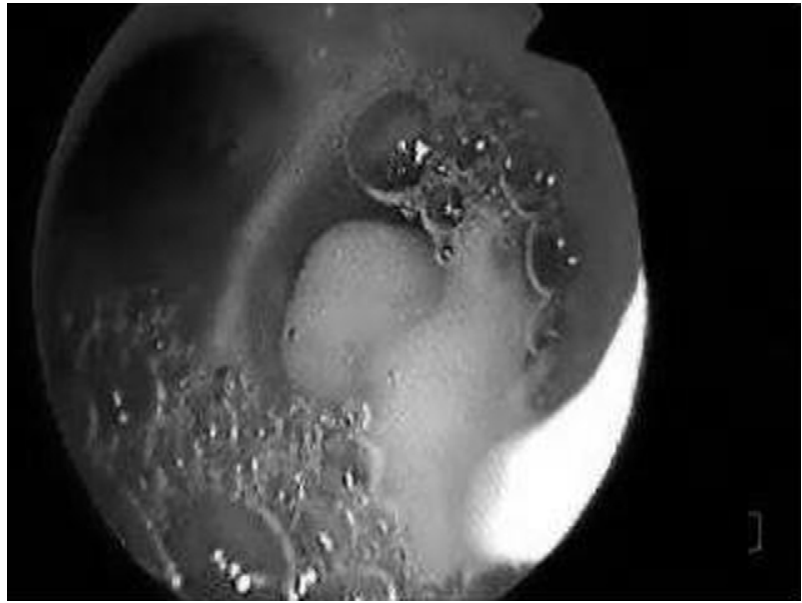

Figura 1 Imagen endoscópica de tapón mucoso central localizado en la entrada del bronquio principal derecho.

seguido de las indicaciones terapéuticas en especial la resolución de atelectasias, con un porcentaje de resolución del $71,4 \%$, principalmente en las causadas por tapón mucoso central. En la figura 1 se muestra imagen endoscópica de un paciente con atelectasia completa de pulmón al que se le aspiró un tapón mucoso central procedente del bronquio principal derecho.

En 27 pacientes (13\%) se detectaron complicaciones menores. Las complicaciones descritas durante el procedimiento fueron taquicardia supraventricular $(3,8 \%)$, hipoxemia transitoria $(6,7 \%)$ y sangrado leve de la mucosa bronquial con el roce del broncoscopio en un 2,4\%. No se ha producido ningún neumotórax en relación con el procedimiento ni necesidad de intubación orotraqueal en los pacientes que no tenían ventilación mecánica en el momento del procedimiento.

\section{Discusión}

El presente estudio describe la utilidad de la fibrobroncoscopia en el enfermo crítico tanto con fines diagnósticos como terapéuticos; ha mostrado ser eficaz, entre otras indicaciones, en el diagnóstico de neumonía y en la resolución de atelectasias. Se trata de un procedimiento seguro, las complicaciones relacionadas con el mismo son menores y se presentan en un porcentaje bajo de casos.

La neumonía en enfermos críticos es la infección nosocomial más frecuente en $\mathrm{UCl}^{8-10}$, conlleva una elevada mortalidad $^{11,12}$, prolongación del tiempo de ventilación mecánica y alto coste económico por un alargamiento de la estancia hospitalaria ${ }^{13,14}$. En nuestra $\mathrm{UCl}$ se aplica la estrategia «invasiva» ${ }^{15-18}$ basada en el uso de fibrobroncoscopia para el diagnóstico microbiológico mediante cultivos cuantitativos de muestras obtenidas selectivamente a nivel de la zona supuestamente afecta. Esta estrategia es recomendada en las guías clínicas de las principales sociedades científicas $^{12,19}$ para el diagnóstico de neumonía asociada a ventilación mecánica (NAV). En nuestra serie, en casi la mitad de los pacientes a los que se les realizó BAL se obtuvo un resultado microbiológico positivo, indicándose principalmente ante la sospecha clínica de neumonía

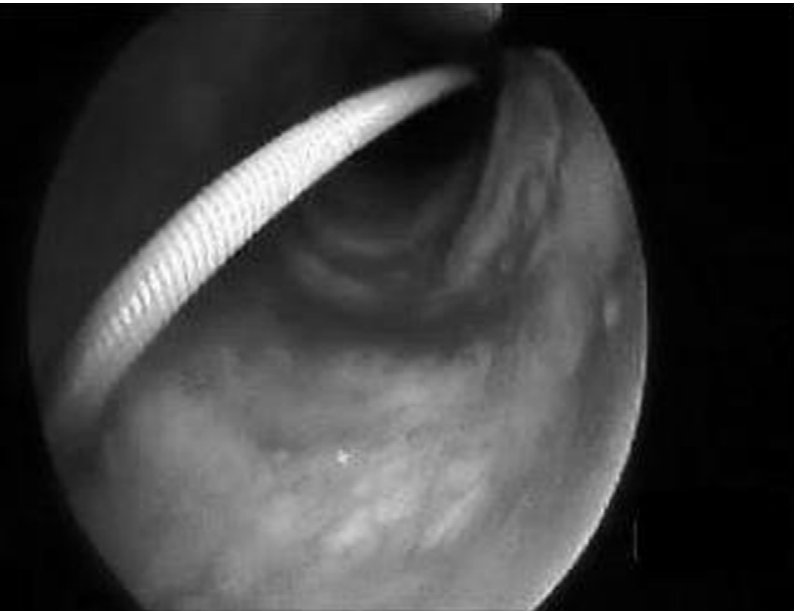

Figura 2 Imagen endoscópica tomada durante la realización de traqueotomía percutánea, momento de introducción de la guía metálica.

asociada a ventilación mecánica o en enfermos inmunodeprimidos en los cuales el BAL ha mostrado tener una mayor rentabilidad ${ }^{20}$. La aparición de atelectasias fue la indicación terapéutica más frecuente. La eficacia de la broncoscopia en su resolución es motivo de controversia. Kreider et al. ${ }^{21}$ en una revisión sistemática de las publicaciones encontraron una importante variación de la eficacia de la fibrobroncoscopia, entre un 19 y un $89 \%^{22,23}$, obteniéndose los mejores resultados en la atelectasia lobar causada por un tapón mucoso central, y los peores en atelectasias subsegmentarias. Marini et al. ${ }^{24}$ compararon la utilización de fibrobroncoscopia frente a fisioterapia respiratoria intensa sin encontrar diferencias entre ambos grupos, condicionando en este estudio que la presencia de broncograma aéreo en la radiología de tórax se asociaba a un mayor retraso en su resolución.

La traqueotomía percutánea ${ }^{25,26}$ es un procedimiento frecuentemente realizado en $\mathrm{UCl}$, con pocas complicaciones pero potencialmente graves como falsa vía, neumotórax, enfisema subcutáneo, fístula traqueoesofágica, etc. estando estas relacionadas principalmente con la falta de visualización de la vía aérea durante la realización de la técnica. Un control broncoscópico minimiza las complicaciones de la técnica ${ }^{27}$, sobre todo en enfermos con cuellos anatómicamente difíciles. Su introducción en estos casos aumenta la seguridad clínica de un procedimiento habitual en $\mathrm{UCl}$. En la figura 2 se muestra el detalle en el que se visualiza la introducción de la guía en la luz traqueal durante una traqueotomía realizada a uno de los pacientes incluidos en el estudio. La disponibilidad del fibrobroncoscopio en la $\mathrm{UCl}$ es fundamental para el abordaje de la denominada «vía aérea difícil» ${ }^{28,29}$, aunque realmente es una indicación poco utilizada ${ }^{5,22}$ debido a que se requiere experiencia para la realización de la técnica. La tercera indicación en orden de frecuencia de la serie presentada fue para el manejo de la hemorragia pulmonar. En esta enfermedad la broncoscopia mostró ser de utilidad no solo diagnóstica sino también con fines terapéuticos durante el procedimiento en casos en los que se objetivan signos de sangrado activo ${ }^{30,31}$. Se han descrito taponamientos del punto de sangrado mediante un catéter de Fogarty ${ }^{32}$ colocado a través del 
canal de aspiración del endoscopio; también se ha documentado la misma técnica descrita pero utilizando un catéter de Swan-Ganz ${ }^{3}$. Aportamos la experiencia de la administración intrapulmonar de factor VII activado instilado a través del canal del broncoscopio en dos emergencias vitales por hemorragia alveolar refractaria a terapia convencional que presentaron una favorable respuesta con este tratamiento. No obstante esta experiencia ha de ser tomada con prudencia ya que son pocos casos los descritos en la literatura y son necesarios futuros estudios que evalúen este tratamiento y avalen su eficacia ${ }^{33,34}$. Otras indicaciones fueron un caso de proteinosis alveolar, revisión de las cuerdas vocales en un caso de ahorcamiento, un caso de ahogamiento en agua de mar en el que se aspiró a través del canal del broncoscopio abundante cantidad de algas y arena, un traumatismo torácico en el que se utilizó la broncoscopia para explorar lesiones del árbol bronquial bajo visión directa ${ }^{35,36}$ y una intubación dirigida con el broncoscopio a través de una prótesis traqueal en la que no fue posible inicialmente intubarlo.

La seguridad de la fibrobroncoscopia ha sido avalada por distintos autores ${ }^{1-5}$. Se presentaron complicaciones en un $13 \%$ de los casos aunque no tuvieron repercusión clínica significativa, por lo que podemos considerar que la técnica es segura en el enfermo crítico siempre y cuando el procedimiento se lleve a cabo en unas condiciones determinadas que garanticen una mayor seguridad clínica. En el apartado pacientes y métodos hemos definido aquellas situaciones en las que no nos parece recomendable realizar el procedimiento al entender que el riesgo supera el beneficio derivado de la técnica insistiendo especialmente en la importancia del diámetro del tubo orotraqueal que ha de ser $2 \mathrm{~mm}$ mayor que el diámetro del broncoscopio, la necesidad de monitorización continua durante el procedimiento y el modo de ventilación; de forma novedosa nuestra experiencia contradice la recomendación clásica citada en la mayoría de los textos de medicina crítica de suspender la PEEP durante el procedimiento ${ }^{37}$. En ninguno de los casos en ventilación mecánica suspendimos la PEEP que tenían los pacientes previamente al procedimiento, basados en la consideración de que el efecto de suspender la PEEP puede agravar la ya comprometida función respiratoria del paciente; es de destacar que no se produjo ningún neumotórax en el modo de ventilación controlada por presión monitorizando estrechamente la presión inspiratoria de acuerdo con las recomendaciones de Lawson et al. que demostraron la consecución de mejores volúmenes respiratorios en presión control que en modo volumen control ${ }^{38}$.

Al tratarse de un estudio retrospectivo, no está exento de limitaciones; la fibrobroncoscopia se realizó por indicación del médico responsable, sin un protocolo previo en el que se definieran las indicaciones, influyendo por tanto, en la selección de la muestra; a pesar de ello, las indicaciones del procedimiento se discuten y acuerdan por el equipo médico en sesión clínica diaria con la intención de minimizar la variabilidad en la práctica clínica. Otra limitación es que se trata de un estudio realizado en un solo centro por lo que consideramos prudente la necesidad de futuros estudios que evalúen nuestras observaciones antes de poder generalizarlas. De igual forma consideramos que también presenta fortalezas; la principal es que esta serie es en la actualidad la más larga documentada en la literatura en enfermos críticos en los últimos años, aportando aspectos novedosos como la seguridad del procedimiento en modo presión control sin necesidad de suspender la PEEP previa. Como corolario de lo anterior y en base a nuestra experiencia consideramos que disponer de fibrobroncoscopia y personal entrenado en su manejo es altamente recomendable en el abordaje de la enfermedad pulmonar en enfermos críticos.

\section{Conflicto de intereses}

Los autores declaran que no tienen ningún conflicto de interés.

\section{Bibliografía}

1. Dellinger RP, Bandi V. Fiberoptic bronchoscopy in the intensive care unit. Crit Care Clin. 1992;8:755-72.

2. Liebler JM, Markin CJ. Fiberoptic bronchoscopy for diagnosis and treatment. Crit Care Clin. 2000;16:83-100.

3. Jolliet Ph, Chevrolet JC. Bronchoscopy in the intensive care unit. Intensive Care Med. 1992;18:160-9.

4. Turner JS, Willcox PA, Hayhurst MD, Potgieter PD. Fiberoptic bronchoscopy in the intensive care unit. A prospective study of 147 procedures in 107 patients. Crit Care Med. 1994;22:259-64.

5. Shennib H, Baslaim G. Bronchoscopy in the intensive care unit. Chest Surg Clin N Am. 1996;6:349-61.

6. British Thoracic Society Guidelines. on diagnostic flexible bronchoscopy. Thorax. 2001;56 Supl 1:1-21.

7. Loanas M, Ferrer R, Angrill J, Ferrer M, Torres A. Microbial investigation in ventilator-associated pneumonia. Eur Respir J. 2001;17:791-801.

8. Richards MJ, Edwards JR, Culver DH, Gaynes RP. Nosocomial infections in combined medical-surgical intensive care units in the United States. Infect Control Hosp Epidemiol. 2000;21:510-5.

9. Vincent JL, Bihari DJ, Suter PM, Bruining HA, White J, Nicolas-Chanion MH, et al. The prevalence of nosocomial infection in Intensive Care Units in Europe. Results of the European Prevalence of Infection in Intensive Care (EPIC) study. EPIC International Advisory Committee. JAMA. 1995;274:639-44.

10. Davis KA. Ventilator-associated pneumonia: a review. J Intensive Care Med. 2006;21:211-26.

11. Moine P, Vercken JB, Chevret S, Chastang C, Gagdos P. Severe community-acquired pneumonia: etiology, epidemiology, and prognosis factors. Chest. 1995;107:1182-3.

12. American Thoracic Society Documents. Guidelines for the management of adults with hospital-acquired, ventilator-associated, and health care-associated pneumonia. Am J Respir Crit Care Med. 2005;171:388-416.

13. The ENVIN-UCl Study GroupOlaechea PM, Ulibarrena MA, Álvarez-Lerma $\mathrm{F}$, Insausti J, Palomar $\mathrm{M}$, de la Cal MA, et al. Factors related to hospital stay among patients with nosocomial infection acquired in the intensive care unit. Infect Control Hosp Epidemiol. 2003;24:207-13.

14. Rello J, Ollendorf DA, Oster G, Vera-Llonch M, Bellm L, Redman R, et al. Epidemiology and outcomes of ventilatorassociated pneumonia in a large US database. Chest. 2002;122:2115-21.

15. Shorr AF, Sherner JH, Jackson WL, Kollef MH. Invasive approaches to the diagnosis of ventilator-associated pneumonia: a meta-analysis. Crit Care Med. 2005;33:46-53.

16. Fagon JY, Chastre J, Wolff M, Gervais C, Parer-Aubas S, Stéphan F. Invasive and noninvasive strategies for management of suspected ventilator-associated pneumonia: a randomized trial. Ann Intern Med. 2000;132:621-30. 
17. Fagon JY. Diagnosis and treatment of ventilator-associated pneumonia: fiberoptic bronchoscopy with bronchoalveolar lavage is essential. Semin Respir Crit Care Med. 2006;27:034-44.

18. Timsit JF, Cheval C, Gachot B, Bruneel F, Wolff M, Carlet J, et al. Usefulness of a strategy based on bronchoscopy with direct examination of bronchoalveolar lavage fluid in the initial antibiotic therapy of suspected ventilator-associated pneumonia. Intensive Care Med. 2001;27:640-7.

19. Álvarez-Lerma F, Álvarez-Sánchez B, Barcenilla F. Protocolo diagnóstico y terapéutico de la neumonía asociada a ventilación mecánica. En: Guía de práctica clínica en medicina intensiva. Sociedad Española de Medicina Intensiva y Unidades Coronarias. Barcelona: Meditex; 1996. p. 1-8.

20. Estella A, Monge MI, Pérez Fontaiña L, Sainz de Baranda A, Galá MJ, Moreno E. Bronchoalveolar lavage for diagnosing pneumonia in mechanically ventilated patients. Med Intensiva. 2008;32:419-23.

21. Kreider MD, Lipson DA. Bronchoscopy for atelectasis in the ICU. A case report and review of the literature. Chest. 2003;124:344-50.

22. Olopade CO, Prakash UB. Bronchoscopy in the critical care unit. Mayo Clin Proc. 1989;64:1255-63.

23. Snow N, Lucas AE. Bronchoscopy in the critically ill surgical patient. Am Surg. 1984;50:441-5.

24. Marini JJ, Pierson DJ, Hudson LD. Acute lobar atelectasis: a prospective comparison of fiberoptic bronchoscopy and respiratory therapy. Am Rev Respir Dis. 1979;119:971-8.

25. Barba CA, Angood PB, Kauder DR, Latenser B, Martin K, McGonigal MD, et al. Bronchoscopic guidance makes percutaneous tracheostomy a safe,cost-effective, and easy-to-teach procedure. Surgery. 1995;118:879-83.

26. Polderman KH, Spijkstra JJ, de Bree R, Christiaans HM, Gelissen HP, Wester JP, et al. Percutaneous dilatational tracheostomy in the ICU: optimal organization, low complication rates, and description of a new complication. Chest. 2003;123:1595-602.
27. Kost KM. Percutaneous tracheostomy: comparison of Ciaglia and Griggs techniques. Critical Care Med. 2000;4:143-6.

28. Butler KH, Clyne B. Management of the difficult airway: alternative airway techniques and adjuncts. Emerg Med Clin North Am. 2003;21:259-89.

29. Ovassapian A. The flexible bronchoscope. A tool for anesthesiologists. Clin Chest Med. 2001;22:281-99.

30. Poe RH, Israel RH, Marin MG, Ortiz CR, Dale RC, Wahl GW, et al. Utility of fiberoptic bronchoscopy in patients with hemoptysis and a nonlocalizing chest roentgenogram. Chest. 1988;93: 70-5.

31. Dupree HJ, Lewejohann JC, Gleiss J, Muhl E, Bruch HP. Fiberoptic bronchoscopy of intubated patients with life-threatening hemoptysis. World J Surg. 2001;25:104-7.

32. Gottlieb LS, Hillberg R. Endobronchial tamponade therapy for intractable hemoptysis. Chest. 1975;67:482-3.

33. Heslet L, Nielsen JD, Levi M, Sengeløv H, Johansson PI. Successful pulmonary administration of activated recombinant factor VII in diffuse alveolar hemorrhage. Crit Care. 2006; 10:R177.

34. Estella A, Jareño A, Perez-Bello Fontaiña L. Intrapulmonary administration of recombinant activated factor VII in diffuse alveolar haemorrhage: a report of two case stories. Cases J. 2008;1:150.

35. Pardon R, Ranic V, Rakaric-Poznanovic M. Bronchoscopy in the diagnosis and therapy of chest injuries. Acta Med Croatica. 1997;51:29-36.

36. Hara KS, Prakash UB. Fiberoptic bronchoscopy in the evaluation of acute chest and upper airway trauma. Chest. 1989;96:627-30.

37. Lindholm CE, Ollman B, Snyder JV, Millen EG, Grenvik A. Cardiorespiratory effects of flexible fiberoptic bronchoscopy in critically ill patients. Chest. 1978;74:362-8.

38. Lawson RW, Peters JI, Shelledy DC. Effects of fiberoptic bronchoscopy during mechanical ventilation in a lung model. Chest. 2000;118:824-31. 